\title{
Fatty Acid-Binding Protein 4 (FABP4) Suppresses Proliferation and Migration of Endometrial Cancer Cells via PI3K/Akt Pathway
}

\author{
Zimeng $\mathrm{Wu}^{\mathrm{I}}$ \\ ji-Hak Jeong ${ }^{2}$ \\ Chenchen Ren' \\ Li Yang' \\ Leilei Ding' \\ Feiyan $\mathrm{Li}^{1}$ \\ Dongyuan Jiang' \\ Yuanhang Zhu' \\ Jie Lu'
}

'Department of Obstetrics and Gynecology, The Third Affiliated Hospital of Zhengzhou University, Zhengzhou, 450052, People's Republic of China; ${ }^{2}$ Research Institute of Pharmaceutical Sciences, College of Pharmacy, Kyungpook National University, Daegu, 41566, Republic of Korea
Correspondence: Chenchen Ren; Li Yang Department of Obstetrics and Gynecology, The Third Affiliated Hospital of Zhengzhou University, No. 7, Front Kangfu Street, Zhengzhou, 450052,

Henan Province, People's Republic of China

Tel/Fax +86-037I-66903982

Email zdsfy_renchenchen@163.com; zdsfyyangli@163.com
Purpose: Endometrial cancer (EC) is the sixth most common cancer in women and its incidence and mortality have been rising over the last decades. The latest research indicates that FABP4 plays a significant role in multiple types of cancer. But few studies were focused on EC. The aim of this article is to investigate whether FABP4 can suppress tumor growth and metastasis of EC via PI3K/Akt pathway to provide a novel therapeutic target for the treatment of EC

Materials and Methods: FABP4 mRNA levels of EC were analysed through The Cancer Genome Atlas database (TCGA), and expression of FABP4 in EC cancer tissues was determined by immunohistochemistry (IHC) assays. Stable overexpressing cell lines were established using lentivirus infection to analyze the biological function of FABP4 in vitro. CCK 8 assay and colony formation assay were performed to assess cell proliferation ability. Wound healing assay and transwell were performed to analyse migration and invasion of cells. The subcutaneous xenograft mouse model was used to evaluate tumor growth in vivo. Additionally, all protein levels were detected by Western blotting assay.

Results: We found that the expression of the FABP4 mRNA was decreased in tumor samples compared to normal tissue according to TCGA database analysis. Subsequent experimental mRNA and protein expression analysis confirmed that FABP4 expression was lower in EC tissue than normal endometrial tissue. In addition, we found overexpression of FABP4 inhibited the proliferation, migration and invasion in vitro and suppressed tumor growth in vivo. Further functional and mechanistic analysis of FABP4 demonstrated that its function is mediated by restraining the phosphorylation of PI3K/Akt signaling pathway.

Conclusion: Our studies shed light for the first time about the functional role of FABP4 in EC and provide a novel biomarker for EC as well as a therapeutic target for the therapy of EC.

Keywords: endometrial cancer, fatty acid-binding protein 4, proliferation, migration, PI3K/ Akt signaling

\section{Introduction}

Endometrial cancer (EC) is a malignancy that originates from the endometrial lining of the uterus, which predominantly occurs in post-menopausal women. ${ }^{1}$ According to the Global Cancer Statistics (GLOBOCAN 2020), EC was the fourth leading cause of gynecological cancer death in the world. The rate of incidence of EC is rapidly increasing, and is expected to increase by more than $50 \%$ worldwide by 2040 (compared to 2018). ${ }^{2,3}$ The causes of this trend could be an increased rate of advanced cancers, high-risk histology, and patients diagnosed at an older age. ${ }^{4,5}$ 
Currently, the most commonly used treatment regimen for EC, worldwide, is surgical adjuvant chemoradiotherapy without effective targeted therapies. Combining chemotherapy with radiation improves the prognosis of EC patients, but usually results in higher toxicity due to their negative effects on normal cells or organs. ${ }^{6}$ Thus, it is necessary to identify specific biomarkers for EC diagnosis, therapy, and prognosis, and to investigate their role in $\mathrm{EC}$ development.

Fatty acid-binding protein 4 (FABP4), also called adipocyte FABP (AFABP) or adipocyte P2 (aP2), is a member of a large gene family encoding small, cytoplasmic fatty acid-binding proteins (FABPs) that is highly expressed in macrophages, adipose tissue and mature adipocytes. ${ }^{7-9}$ Previous studies showed that FABP4 is mainly involved in metabolic homeostasis and inflammatory diseases. However, recent studies have suggested that it is also implicated in the progression of various cancers, such as ovarian cancer, cervical cancer, and breast cancer. ${ }^{8,10-13}$ Therefore, the therapeutic targeting of FABP4 in tumors has attracted increasing interest in the scientific community and understanding its precise role is an important challenge that deserves further research.

Although the association of FABP4 with other tumors has been previously demonstrated, the precise role of FABP4 in EC, and its mechanism of action remain unclear. We demonstrated that FABP4 could effectively suppress EC cells growth both in vitro and in vivo through the PI3K/Akt signaling pathway. Therefore, our study characterizes the biological function of FABP4 in EC and identifies a potential new target for possible EC therapy and prognostic evaluation of EC patients.

\section{Materials and Methods}

\section{Cell Culture and Transfections}

The human EC cell lines HEC-1A and KLE were purchased from the Beina Chuanglian Biotechnology Institute (Beijing, China), and Procell Life Science \& Technology Co., Ltd. (Wuhan, China), respectively. The Ishikawa cell line was a gift from the Department of Obstetrics and Gynecology, First Affiliated Hospital of Zhengzhou University. The use of the Ishikawa cell line had been approved by the Ethics Committee of the Third Affiliated Hospital of Zhengzhou University (Protocol \# 2021-WZ -013). The HEC-1A cells were cultured in Mccoy's 5A medium (Procell Life Science \& Technology Co., Ltd.), the Ishikawa and KLE cells were cultured in Dulbecco's modified Eagle's medium (DMEM; Gibco, Thermo Fisher Scientific, Inc., Waltham, MA, USA). All cell lines were cultured in their corresponding medium containing $10 \%$ of fetal bovine serum (FBS; Gibco, Thermo Fisher Scientific, Inc.) and penicillin-streptomycin at $37^{\circ} \mathrm{C}$, with $5 \% \mathrm{CO}_{2}$. Then, the two low FABP4-expressing cell lines were chosen for lentivirus transfection. The FABP4 lentiviral vector used in this process was the G260 vector (Ubi-MCS-firefly _Luciferase-IRES-Puromycin; GeneChem, Shanghai, China), which can produce luciferase for in vivo imaging. Cells were grown to $20-30 \%$ confluence in 6-well plates and then transfected with the lentiviral vector. Monoclonal cell lines were screened with $0.25 \mu \mathrm{g} / \mathrm{mL}$ puromycin, and the clones with the highest FABP4 expression levels, as determined by quantitative real-time polymerase chain reaction (qRT-PCR), were retained for subsequent cellular experiments.

\section{Tissue Samples}

Tissue specimens were obtained from surgical resection of EC performed in the Department of Pathology, of The Third Affiliated Hospital of Zhengzhou University (Zhengzhou, China). They included 50 EC specimens, 38 atypical hyperplasia specimens and 29 normal endometrium specimens. None of the patients received chemotherapy or radiotherapy before operation. The study was approved by the Medical Ethics Committee of the Third Affiliated Hospital of Zhengzhou University (ethics approval number: 2019-64) and complies with the Declaration of Helsinki. All patients provided informed written consent prior to enrollment in this study.

\section{The Cancer Genome Atlas (TCGA) Datasets}

The EC raw data, including clinicopathological parameters and FABP4 mRNA expression level, were downloaded from TCGA to analyze the difference in mRNA expression level and perform further survival curve analysis.

\section{Immunohistochemistry (IHC) Staining and Analysis}

A standard biotin-streptavidin horse radish peroxidase (HRP) detection system was used for visualization of immunostained protein. Paraffin-embedded tissues were first cut into $5-\mu \mathrm{m}$ thick sections. Then, after a series of steps, including dewaxing and hydration, antigen repair, and blocking endogenous peroxidase, the sections were 
immunoreacted with an FABP4 rabbit monoclonal antibody (ab92501; Abcam, Cambridge, UK), by incubating overnight. Afterwards, the sections were washed with sterile phosphate-buffered saline (PBS) and incubated with rabbit anti-mouse biotin-bound secondary antibody. The FABP4-immunoreacted sections were observed under a microscope and interpreted by qualified pathologists, staining scoring criteria were as previously described. ${ }^{14}$ Tissue sections with a final staining score $\geq 3$ were considered to be positive.

\section{Western Blot Analysis}

Tissue proteins were extracted using RIPA lysis buffer solution (Solarbio, Beijing, China) containing phenylmethylsulfonyl fluoride (PMSF) at a ratio of 100:1. After incubation on ice for $30 \mathrm{~min}$, the cell lysate was centrifuged at $14,000 \mathrm{~g}$ for $15 \mathrm{~min}$ and the supernatant containing the proteins were collected. Protein extracts were separated by sodium dodecyl sulfate-polyacrylamide gel electrophoresis (SDS-PAGE), and transferred to polyvinylidene fluoride (PVDF) membranes (Millipore Corporation, Billerica, MA, USA). Then, after blocking the membranes with skim milk (5\%), the membrane blots were incubated at $4{ }^{\circ} \mathrm{C}$ overnight with primary antibodies: FABP4 (ab92501, 1:2,000; Abcam), GADPH (60004-1-Ig, 1:2,000; Proteintech, Rosemont, IL, USA), PI3K (602251-Ig, 1:1,000; Proteintech), p-PI3K (AF3241, 1:1,000; Affinity Biosciences Ltd., Cincinnati, OH, USA), Akt (10176-2-AP, 1:1000; Proteintech), p-Akt (ab38449, 1:1000, Abcam). GAPDH was used as an endogenous control. Subsequently, the membranes were incubated with the HRP-conjugated secondary antibody for $1 \mathrm{~h}$ at room temperature. Immunoreacted protein bands were detected with the NcmECL Ultra detection system (New Cell \& Molecular Biotech. Co., Ltd., Suzhou, China) on a Bio-Rad Chemi-Doc XRS system (Bio-Rad, Hercules, CA, USA) with automatic exposure control.

\section{qRT-PCR Analysis}

The total RNA was extracted from cells grown $80 \%$ confluence in a six-well plate using $1 \mathrm{~mL}$ Trizol reagent (Invitrogen, Carlsbad, CA, USA), following the manufacturer's protocol. Then, the total RNA was reversed transcribed into cDNA using a reverse transcription kit (Takara, Dalian, China). The qRT-PCR was performed on an ABI 7500 real-time PCR system (Applied Biosystems, Foster City, CA, USA). The $\beta$-actin gene was used as internal reference and the $2^{-\Delta \Delta C T}$ method was used to analyze the data and determine expression levels. ${ }^{15}$ The sequences of the primers for $\beta$-actin were $5^{\prime}$-CACCA TTGGC AATGA GCGGTTC-3' (forward) and 5'AGGTC TTTGC GGATG TCCACGT-3' (reverse). The FABP4 primers were 5'-ACTGG GCCAG GAATT TGACG-3' (forward) and 5'-CTCGT GGAAG TGACG CCTT $-3^{\prime}$ (reverse).

\section{Cell Counting Kit-8 (CCK-8) Assay}

The cell viability was determined by CCK-8 assays. Cells were plated into 96-well plates at a density of $2 \times 10^{3}$ cells/ well and incubated for 1, 2, 3 or 4 days before the addition of CCK-8 solution (Dojindo Molecular Technologies, Inc., Kumamoto, Japan). Then, the incubation was continued under the same conditions for another $2 \mathrm{~h}$. The absorbance or optical density (OD) value of each well was measured at $450 \mathrm{~nm}$ using a Varioskan Flash microplate reader (Thermo Fisher Scientific Inc.).

\section{Colony Formation Assay}

For clonal experiments, $1 \times 10^{3}$ cells were plated into 6 -well plates and cultured for 2 weeks at $37^{\circ} \mathrm{C}$ with $5 \%$ $\mathrm{CO}_{2}$, replacing the medium every 3 days. After fixation with methanol for $10 \mathrm{~min}$, the colonies were stained with $0.1 \%$ crystal violet for $15 \mathrm{~min}$, and colonies ( $\geq 50$ cells) were counted and photographed with a digital camera.

\section{Transwell Migration and Invasion Assay}

For the migration assays, a $100-\mu \mathrm{L}$ aliquot of a cell suspension in serum-free medium was added to the upper chambers, and $600 \mu \mathrm{L}$ of medium with $15 \%$ FBS was added to the lower chamber. After culturing for $24 \mathrm{~h}$, the cells were fixed in 4\% paraformaldehyde and stained with $1 \%$ crystal violet. Then, samples were washed with PBS, visualized and photographed under an Olympus BX51 light microscope (Olympus Corporation, Tokyo, Japan). For the invasion assays, the only difference was that the $100-\mu \mathrm{L}$ aliquot of cell suspension in serum-free medium was added to the apical chamber of the Transwell covered with Matrigel matrix glue (Corning Inc., Cambridge, MA, USA).

\section{Wound Healing Assay}

The wound healing assay was performed in 6 well-plates, $1 \times 10^{6}$ cells were plated into 6 -well plates overnight until the cells reached a confluence of 90-95\%. Then, scratch wounds were made by scratching a straight line in the cell layer with a sterile $200-\mu \mathrm{L}$ pipette tip. At $0,24,48,72$, and 
$96 \mathrm{~h}$, plates were photographed to monitor the wound healing process at those time points. The wound healing area was quantified using the ImageJ software (NIH, Bethesda, MD, USA), and the percentage of wound healing was expressed as the ratio of the original wound area that had healed.

\section{In vivo Subcutaneous Tumor Xenograft Model}

Ethical approval for the animal study was granted by the Ethics Committee for Animal Research of Zhengzhou University (ethics approval number: ZZU-LAC20201023 [04]). Animal experiments adhered to the Guide for the Care and Use of Laboratory Animals. Female nude mice (BALB/c, 3-4 weeks old) were purchased from SPF Biotechnology Co., Ltd. (Beijing, China). Ishikawa cells stably transfected with FABP4-overexpressing lentivirus and control lentivirus were implanted in the right flanks of nude mice $\left(2 \times 10^{6}\right.$ cells, 5 mice per group). Mice were anesthetized with isoflurane, and D-luciferin $(15 \mathrm{mg} / \mathrm{mL}$; US Everbright Inc., Suzhou, China) was injected into each mouse at a dose of $10 \mu \mathrm{L} / \mathrm{g}$. The fluorescence signal intensity of the tumor was determined weekly using the IVIS Living Image software v4.3.1 (Caliper Life Sciences Inc., Hopkinton, MA, USA), and at the same time, the tumor volume was determined with a Vernier caliper. Each tumor volume was separately calculated using the formula of volume $=(\text { width })^{2} \times$ length $/ 2$. The nude mice were sacrificed after 4 weeks and tumors were removed and weighed. Excised tumor tissues were used for Western blot analysis.

\section{Statistical Analysis}

Data were analyzed using the SPSS software (IBM Corporation, Armonk, NY, USA) and Graphpad Prism 8.0 (GraphPad Software Inc., San Diego, CA, USA). All the experimental data were collected from at least three repeated experiments, if not otherwise stated. The data are presented as the mean $\pm \mathrm{SD}$ and the $\mathrm{p}$-value $<0.05$ was considered to indicate statistically significant difference.

\section{Results}

\section{FABP4 is Downregulated and Correlates with High Survival Rate in EC Patients}

To investigate FABP4 expression in EC, we downloaded the EC raw data and gene expression data of FABP4 from TCGA database (https://tcga-data.nci.nih.gov/tcga). The mining of the original data showed that FABP4 was downregulated in
EC tissue compared with normal tissues ( $\mathrm{p}<0.05$; Figure 1A). We assessed the relationship between FABP4 mRNA expression levels and clinicopathological parameters using the clinical staging system proposed by the International Federation of Gynecology and Obstetrics (FIGO). In the FIGO system, "G stage" represents tumor differentiation and higher grades mean lower differentiation and higher malignancy degree; advanced tumor is expressed with higher clinical stage; the "Endometrioid" represents endometrioid endometrial adenocarcinoma, while the 'Serous' represents serous endometrial adenocarcinoma, the latter shows a higher degree of malignancy than the former; the "M" refers to the distant metastasis of tumor cells; the "Pre-", "Peri-" and "Post-", denote female premenopausal, perimenopausal and postmenopausal status, respectively.

Using univariate analyses of TCGA data set, some clinical factors, including age, $\mathrm{G}$ stage, clinical stage, histological types, distant metastasis and menopause status, were significantly associated with FABP4 expression levels (Figure 1B-G). The overall survival (OS) and relapse-free survival (RFS) were estimated using the Kaplan-Meier method. The results revealed that patients with high expression of FABP4 have a better prognosis $(p=0.016$, Figure $1 \mathrm{H}$; $p=0.024$, Figure $1 \mathrm{I}$ ). We then performed multivariate analysis for further analysis on the relationship between FABP4 expression and clinicpathological parameters. Results revealed that reduced FABP4 expression in EC was significantly associated with high $\mathrm{G}$ stage $(\mathrm{OR}=1.88$, Figure 2, $\mathrm{p}=0.002)$, histological types $(\mathrm{OR}=1.96$, Figure $2, \mathrm{p}=0.007)$ and distant metastasis $(\mathrm{OR}=3.64$, Figure 2, $\mathrm{p}=0.028)$. This suggested that ECs with low FABP4 expression are prone to progress to a more advanced stage and distant metastasis than those with high FABP4 expression.

\section{Expression of FABP4 Was Decreased in Human Endometrial Tumor Tissues}

The IHC analysis results showed that the intensity of the staining of the FABP4 protein decreased gradually during the tumor progression process. FABP4 was highly expressed in the normal proliferative phase of the endometrium (Figure $3 \mathrm{~A}$ and B) and normal secretory phase of the endometrium (Figure 3C and D), whereas the staining intensity was significantly reduced in atypical hyperplasia tissues (Figure $3 \mathrm{E}$ and $\mathrm{F}$ ). FABP4 was almost not expressed in EC tissues (Figure 3G and $\mathrm{H})$. All three differences were statistically significant $(\mathrm{P}<$ 0.05; Figure 3I). These results suggested that FABP4 might have a possible tumor-suppressive role in EC. 
A

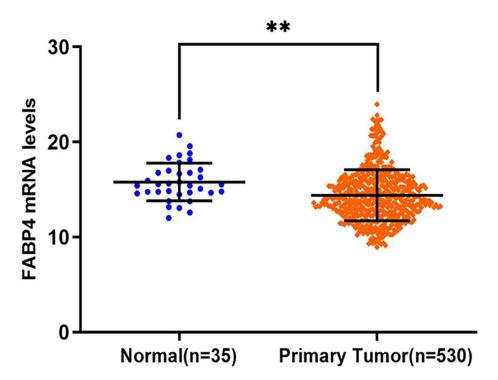

D

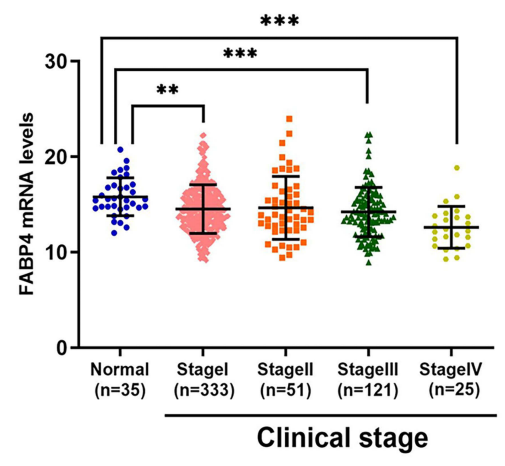

G

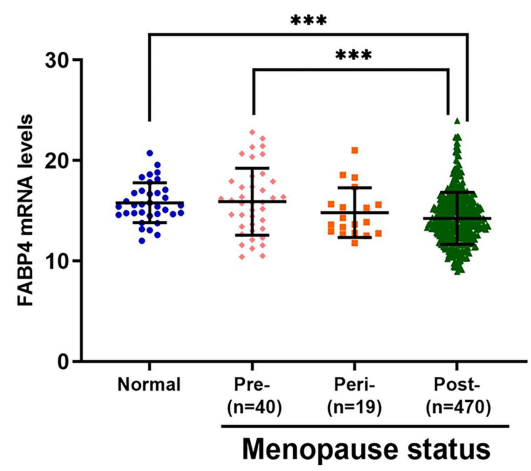

B

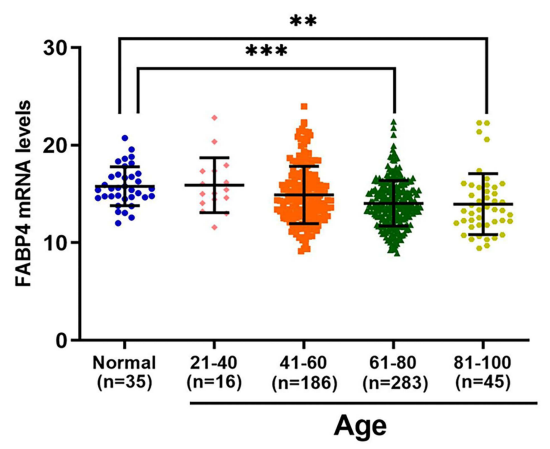

E

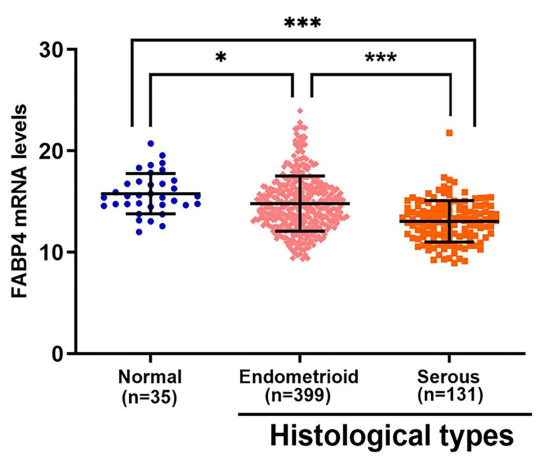

H

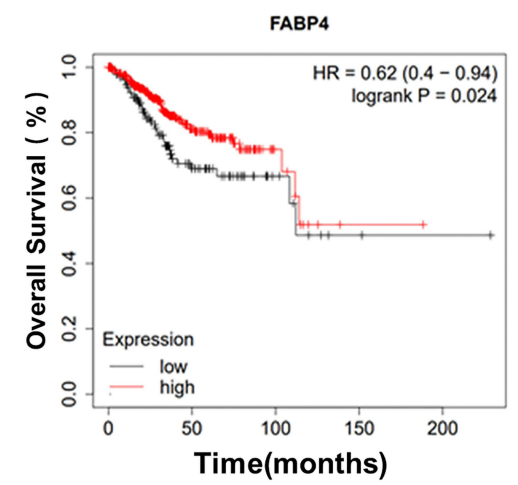

C

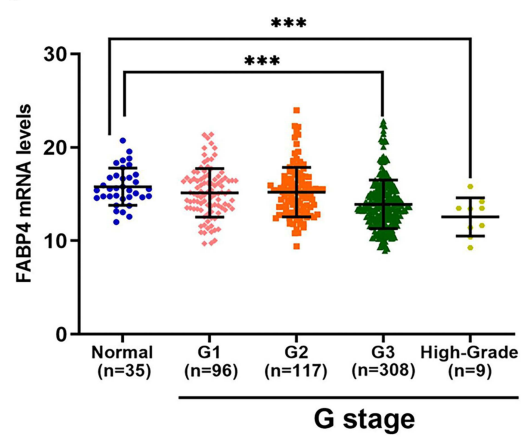

F

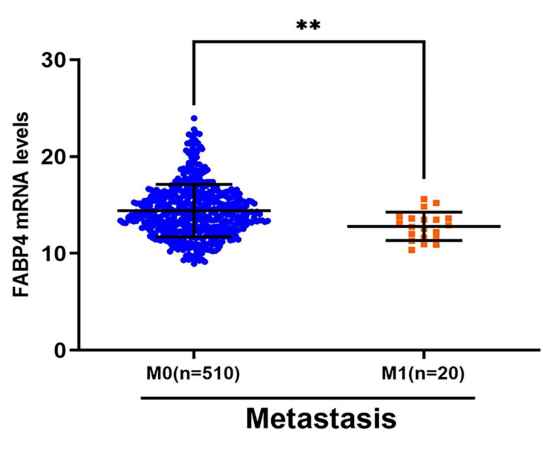

I

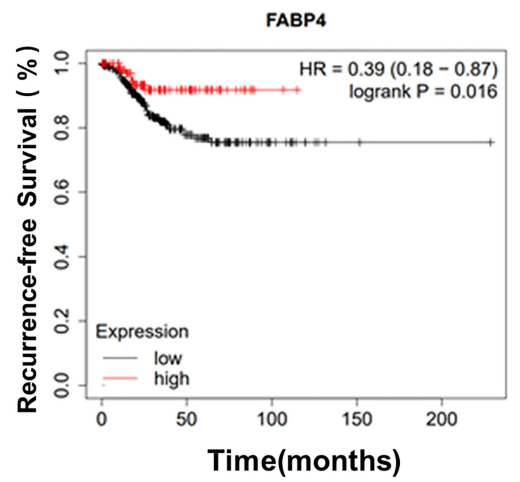

Figure I Clinicopathological correlation between FABP4 and EC. (A) The FABP4 mRNA expression level is downregulated in tumor samples. The FABP4 mRNA expression levels were analyzed together with the following clinical pathological parameters: (B) age, (C) G stage, (D) clinical stage, (E) histological subtypes, (F) metastasis, (G) menopause status. (H and I) Kaplan-Meier survival curve analysis based on TCGA datasets with different FABP4 expression levels. Non-significant comparisons are not shown. Data are shown as the mean $\pm \mathrm{SD}$. ***P $<0.001$, **P $<0.01$, $* \mathrm{P}<0.05$.

Abbreviations: Endometrioid, endometrioid endometrial adenocarcinoma; Serous, serous endometrial adenocarcinoma; “Pre-", "Peri-" and “Post-", female premenopausal, perimenopausal and postmenopausal status, respectively.

\section{FABP4 Suppresses the Proliferation of EC Cell}

Uncontrolled proliferation is one of the most critical characteristics of cancer cells associated with tumor progression. Accordingly, we investigated the biological function of FABP4 in EC cells in vitro. We used 3 different EC cell lines to analyze their FABP4 mRNA and protein expression levels by qRT-PCR analysis (Figure 4A) and Western blot analysis (Figure 4B), respectively. Since HEC-1A and Ishikawa cells showed lower expression of FABP4 than KLE, they were used to establish stably FABP4-overexpressing cell lines using an FABP4 lentivirus. The efficiency of lentiviral transfection in 
FABP4 expression associated with clinical pathological characteristics (logistic regression).

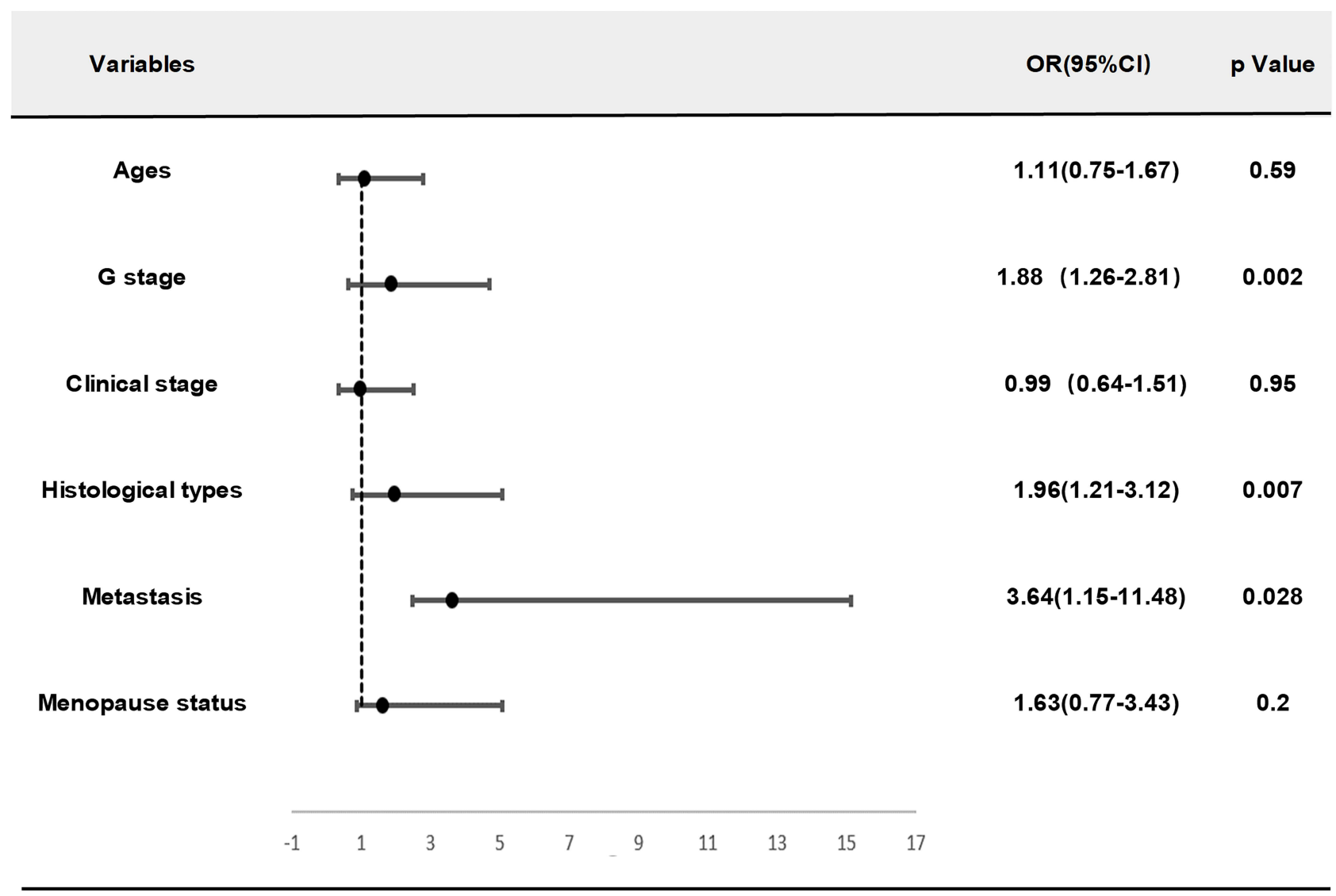

Categorical dependent variable, greater or less than the median expression level.

Figure 2 Multivariate logistic analysis of FABP4 expression and other clinical pathological factors (as G stage, histological types and distant metastasis are independent factors).

two EC cell lines is shown in Figure 4C-F, which indicates that the transfection efficiency is high in both cell lines. Noteworthy, the transfection efficiency was quite high and similar in both cell lines. Using these EC cell lines, we investigated the influence of FABP4 on cell proliferation using the CCK-8 assay. The results indicated that overexpression of FABP4 suppresses cell proliferation (Figure 4G and H). In addition, the results of the colony formation assay demonstrated that FABP4 reduces the clonogenic capacity of EC cell lines (Figure 4I and J).

\section{FABP4 Suppresses the Migration and Invasion of EC Cells in vitro}

To evaluate the effects of FABP4 expression on the metastatic ability of EC cells, we conducted migration and invasion experiments. The number of migrated and invading cells was significantly decreased in FABP4overexpressing cell lines (Figure 5A-D). These results indicated that overexpression of FABP4 inhibits EC cell migration and invasion.

\section{FABP4 Suppresses Tumor Growth in Mouse Models}

To investigate the role of FABP4 in vivo, we examined the role of FABP4 on tumor formation and progression in an experimental xenograft tumor model. The Ishikawa cells stably overexpressing FABP4 were implanted subcutaneously in the right armpit region of nude mice $(n=5)$ and tumors were collected and weighed at 28 days after tumor cell transplantation. The luciferase activity and tumor volume were decreased in FABP4-overexpressing mice (Figure 6A-C), and the tumor weight was significantly decreased in FABP4-overexpressing mice 
A

B

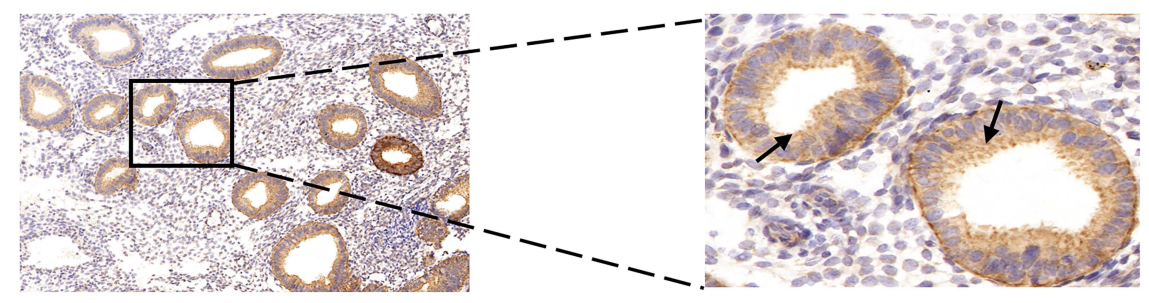

C

D

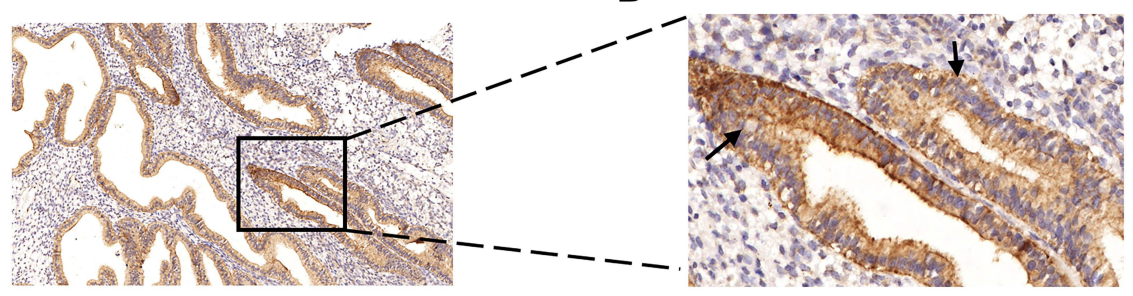

E

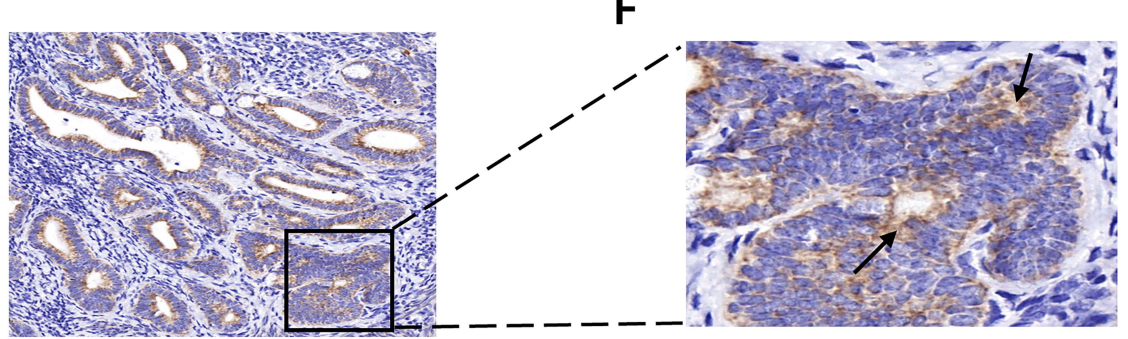

G

H

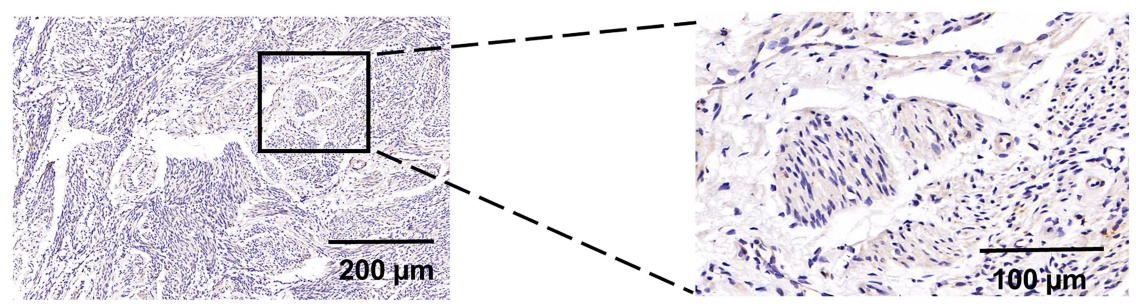

I

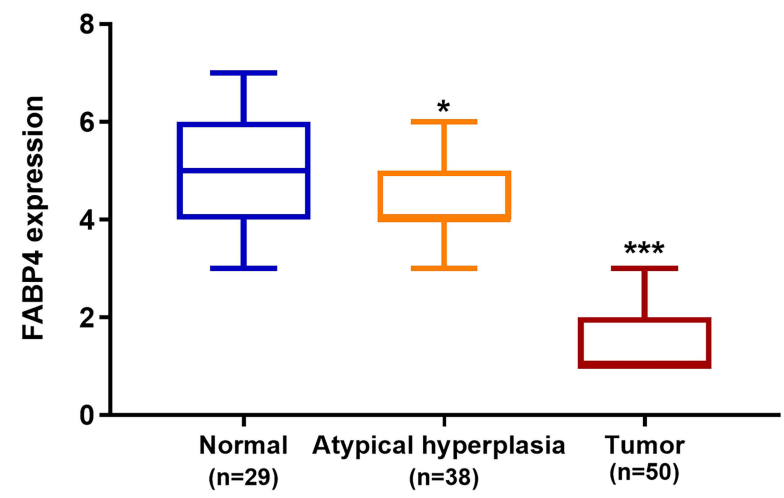

Figure 3 Expression of FABP4 in human endometrial tissues. (A) Normal proliferative stage endometrium, (C) Normal secretory phase endometrium, (E) Atypical hyperplasia endometrium (G) carcinoma. (B, D, F and $\mathbf{H}$ ) are their corresponding enlarged images. (I) Immunohistochemical intensity scores of FABP4 staining in II7 specimens. Data are shown as the mean $\pm \mathrm{SD}$. $* * * \mathrm{P}<0.00 \mathrm{I}$, $* * \mathrm{P}<0.0 \mathrm{I}, * \mathrm{P}<0.05$. 
A

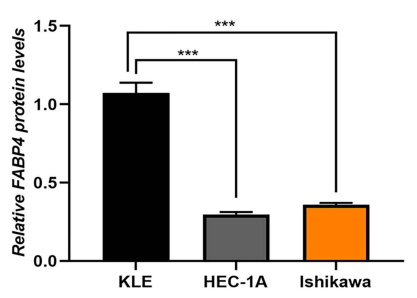

C

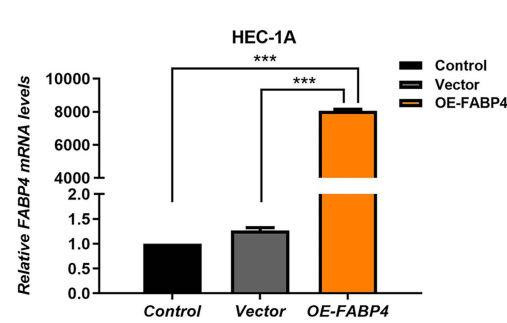

E

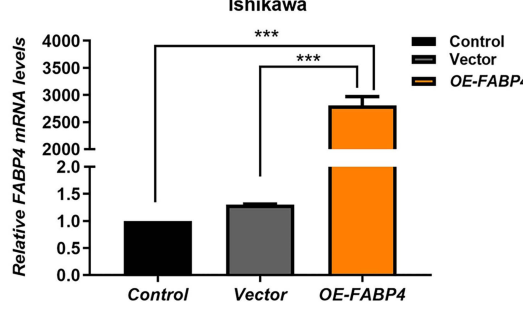

G

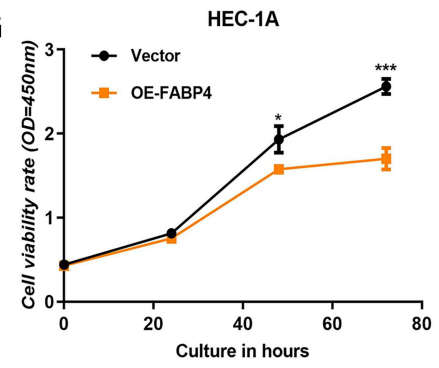

I
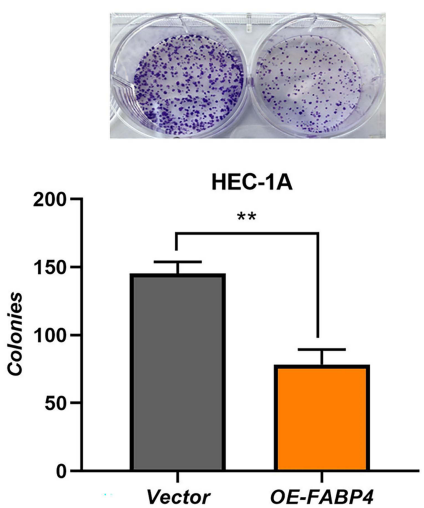

B

FABP4

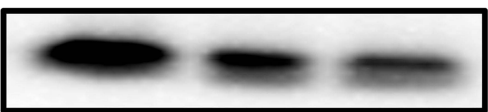

GADPH

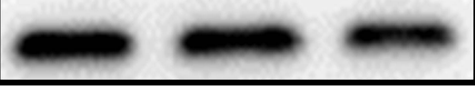

KLE HEC-1A Ishikawa

D

HEC-1A

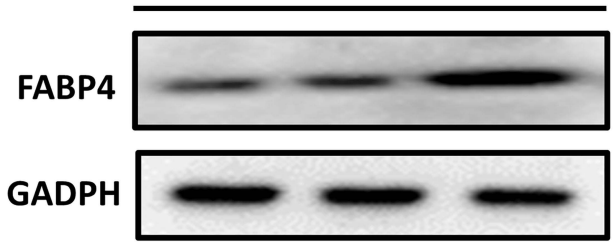

control vector OE-FABP4

F

Ishikawa

FABP4

GADPH

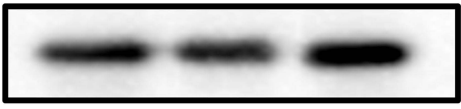

control vector OE-FABP4

H

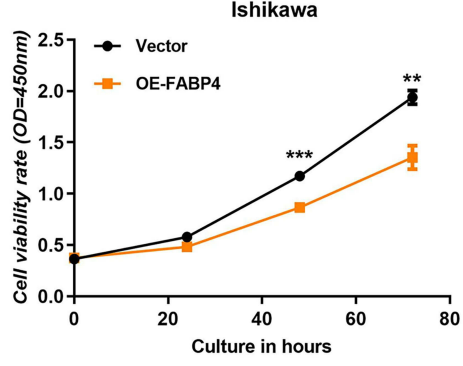

J

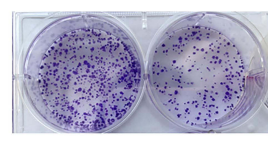

Ishikawa

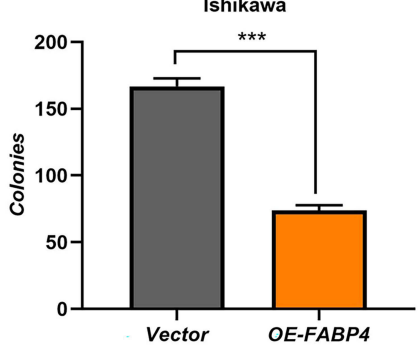

Figure 4 Overexpression of FABP4 inhibits cell proliferation in vitro. FABP4 protein expression levels (A) and mRNA expression levels (B) in endometrial carcinoma cell lines (Ishikawa, HEC-IA, and KLE). (C-F) Identification of two low FABP4-expressing cell lines (Ishikawa, HEC-IA) lentiviral transfection efficiency by qRT-PCR analysis and Western blot analysis. ( $\mathbf{G}$ and $\mathbf{H}$ ) Cell viability was assessed by the CCK-8 assay. (I and J) Colony formation of EC cells assay was performed to evaluate cells proliferation. Data are expressed as the mean $\pm \mathrm{SD}$. $* * * \mathrm{P}<0.00$ I, $* * \mathrm{P}<0.0$ I, $* \mathrm{P}<0.05$. 
A

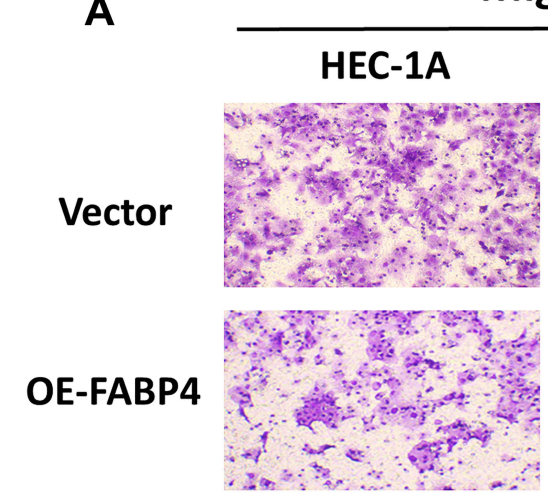

Migration

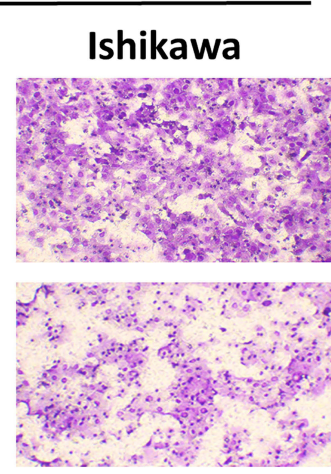

\section{Invasion}

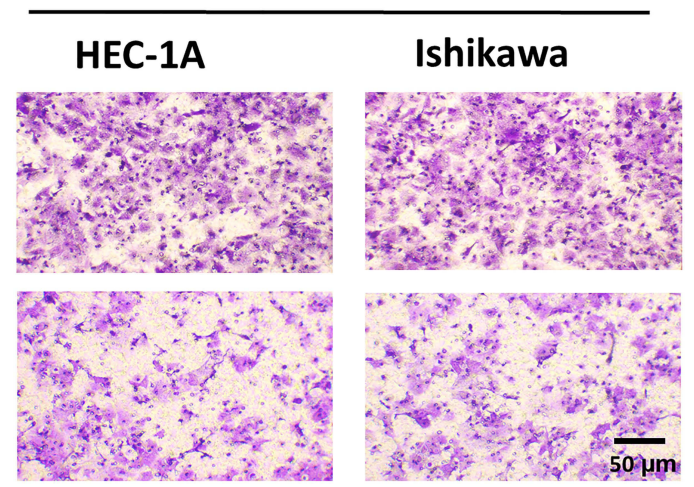

B
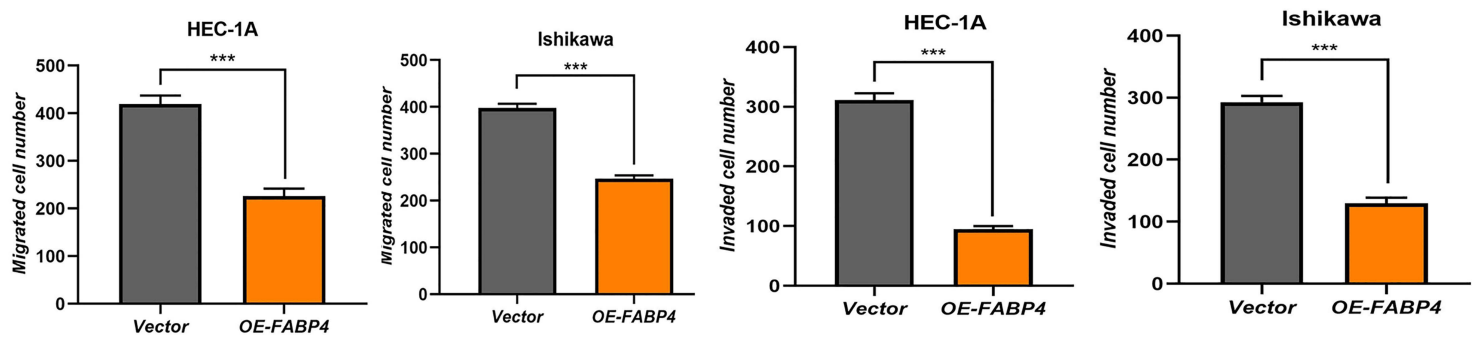

C

HEC-1A
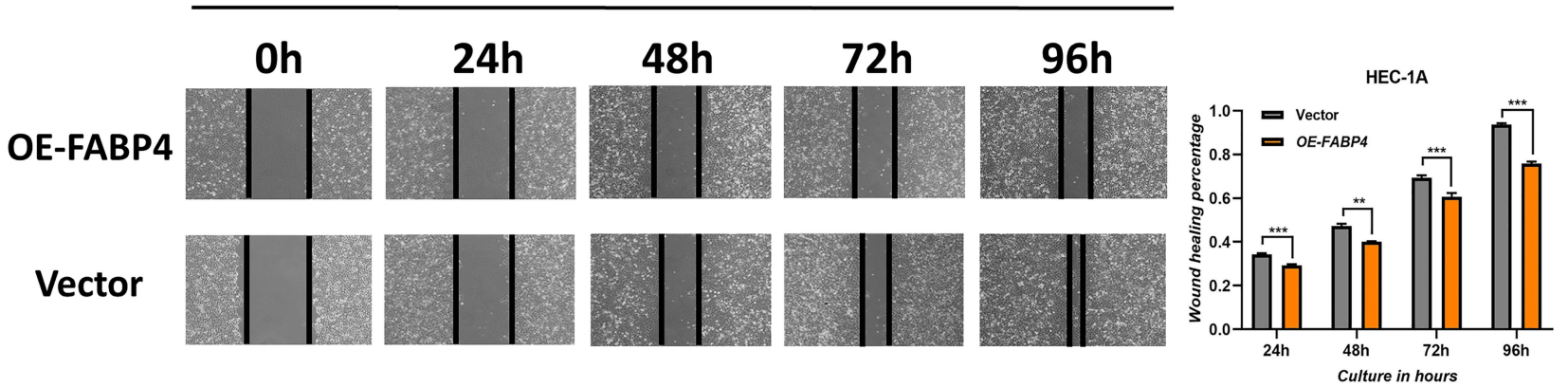

D

Ishikawa

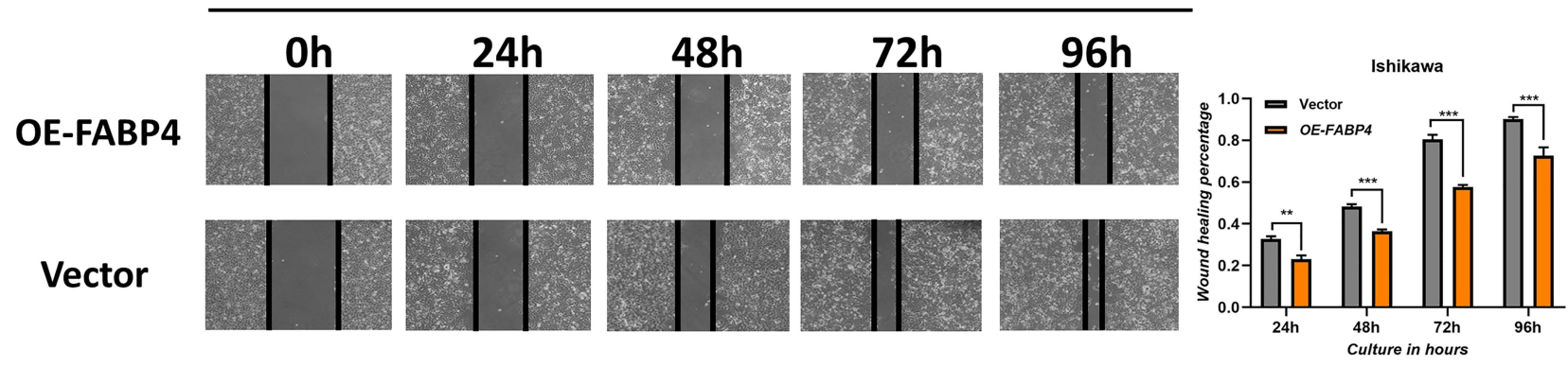

Figure 5 Overexpression of FABP4 inhibits cell migration and invasion in vitro. (A) Transwell migration and invasion assays were performed with FABP4-overexpressing EC cells. (B) Quantification of the Transwell migration and invasion assay results. (C and D) Wound-healing assays indicating impaired migration capability of FBP4overexpressing EC cells, the bar graphs represent quantification of the wound-healing assay results. Data are shown as the mean $\pm \mathrm{SD}$. $* * * P<0.00 \mathrm{I}, * * \mathrm{P}<0.0 \mathrm{I}, * \mathrm{P}<0.05$. 
(Figure 6D and E). These results indicated that overexpression of FABP4 also has EC tumor-suppressive roles in vivo.

\section{FABP4 Inhibits the Activation of PI3K/ Akt in EC}

The PI3K/Akt signaling pathway, which is often aberrantly activated in EC, has been shown to regulate cell proliferation, differentiation and apoptosis, and to be regulated by FABPs in other cancers. ${ }^{30,31}$ Therefore, we investigated whether FABP4 inhibits the development of EC by inhibiting the activation of the PI3K/Akt signaling pathway. As shown in Figure 6, overexpression of FABP4 in EC cells suppressed phosphorylation of PI3K/Akt and thereby their signaling activity (Figure 7A and $\mathrm{B}$ ). This finding was also confirmed in controlled animal experiments (Figure 7C). Overall, these results indicated that FABP4 may suppress the PI3K/Akt signaling pathway by decreasing the phosphorylation of PI3K and Akt.

\section{Discussions}

$\mathrm{EC}$ is one of the most common gynecological malignancy and the fourth most prevalent cancer in women worldwide. ${ }^{16}$ As the incidence and mortality of EC continue to increase, the burden of EC continues to increase compared to various cancers. ${ }^{17}$ Although surgery is the mainstay of the treatment of EC, there are still $25 \%$ of the patients who are in an advanced stage of cancer at the time of surgery, with an expected 5-year survival rate of 40 $70 \%$ for stage III patients, and an extremely poor 5 -year survival rate of $0-10 \%$ for stage IV patients. ${ }^{18}$ With the advances in molecular biology technology and research on signal transduction pathways, biomarker-guided cancer treatments have brought a new breakthrough in prognosis, diagnosis, and therapeutic treatment. For instance, in breast cancer, HER2-targeted therapy has been used clinically to improve the survival of patients with both earlystage and metastatic breast cancers. ${ }^{19,20}$ In lung cancer and hematologic malignancies, biomarker-guided management has been routinely used for patients with advanced or recurrent cancer. ${ }^{21,22}$ However, biomarker-guided treatment of EC is in its infancy and remains largely unexplored. Therefore, it is necessary to find new therapeutic targets to advance the development of treatments and theranostics for EC.

FABPs are a family of intracellular lipid chaperones with at least 12 members, which have been identified since its first member was initially discovered in $1972 .{ }^{23}$ Although these isoforms differ in various tissues, they all perform significant biological functions. ${ }^{7}$ FABP4 is one of the most widely studied FABPs, the function of FABP4 in tumors is extremely complex and sometimes contradictory. Nieman et al reported increased FABP4 expression in ovarian cancer omental metastases, but not in primary tumors. ${ }^{24}$ In cholangiocarcinoma, FABP4 overexpression was found to enhance the intracellular lipid accumulation and promote cholangiocarcinoma cells invasion and metastasis. ${ }^{25}$ Conversely, some studies found that FABP4 was significantly decreased in tumor cells compared with normal prostate epithelial cells and overexpression of FABP4 could induce apoptosis of prostate cancer cells by increasing tumor necrosis factor and decreasing transforming growth factor-alpha activity. ${ }^{26-28}$ Similar results were reported in hepatocellular carcinoma (HCC). For instance, Zhong et al, found that FABP4-overexpressing HCC cell lines exhibited obvious decrease in cell proliferation. ${ }^{29}$ These findings indicate that FABP4 may play different roles depending on the tumor type. A possible explanation for such discrepancies is that FABP4 might play multi-dimensional roles by participating in the regulation of various signaling pathways.

To further explore the biological role of FABP4 in EC, we obtained the clinical data from TCGA. We found that FABP4 was markedly downregulated in EC tissues compared to the corresponding adjacent non-cancerous tissue. Moreover, high expression of FABP4 was negatively associated with $\mathrm{G}$ stage, distant metastasis, and worse pathological types, whereas it was positively correlated with better OS. We also confirmed these results at the tissue level. The IHC results showed that the staining intensity of FABP4 protein gradually decreased in normal endometrial tissues, dysplasia tissues and cancer tissues. These results suggested that FABP4 may have a key role in the progression of EC. To test this assumption, we designed and conducted a series of in vitro and in vivo experiments. The results of the in vitro assays showed that FABP4 effectively inhibited the proliferation, growth, migration and invasion of EC cells. The in vivo studies, in the nude mouse model, revealed that overexpression of FABP4 led to a decrease of tumor size compared to mock-transfected cells. These results are consistent with the effects of FABP4 on liver tumor growth. $^{29}$

Further research is required to gain a better understanding the potential underlying mechanism by which FABP4 influences EC development. The PI3K/Akt 
A

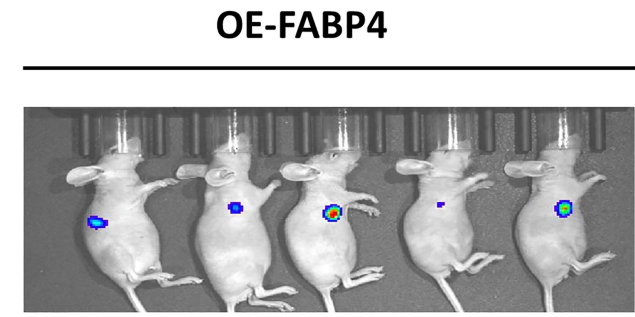

28d

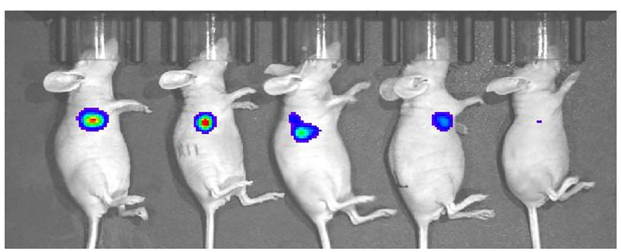

B

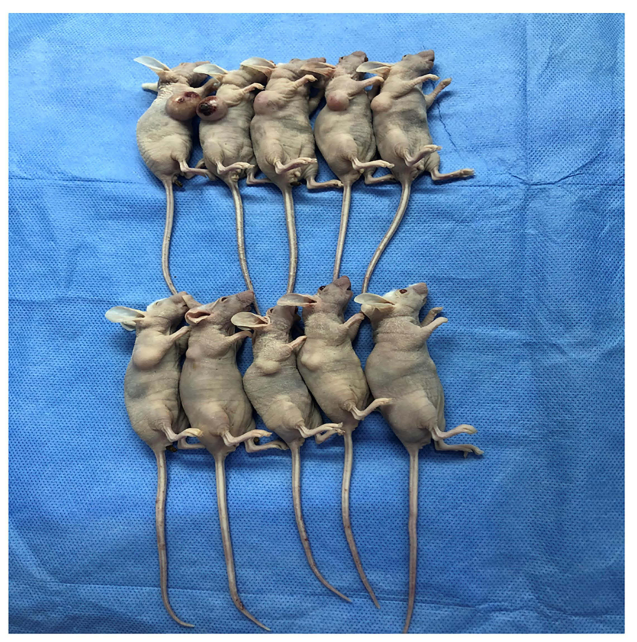

D

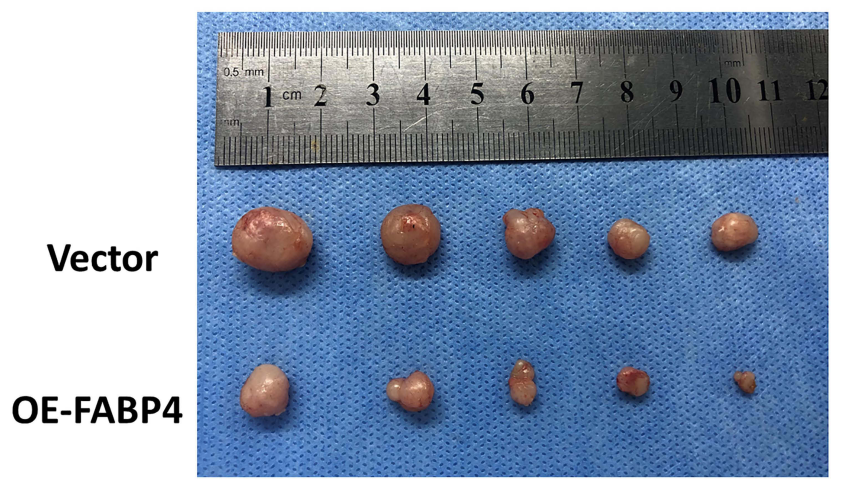

\section{Vector}
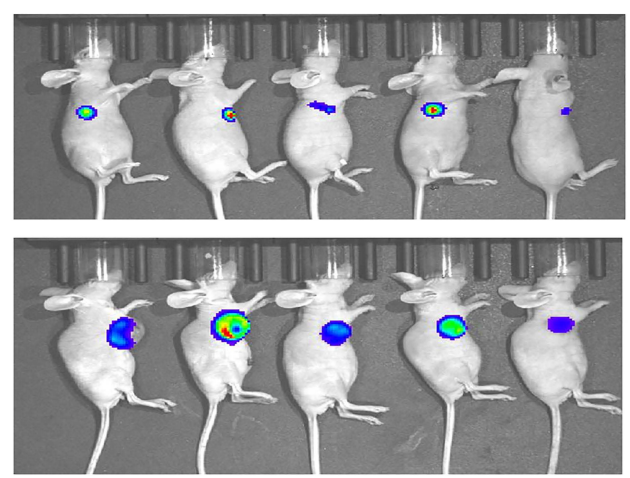

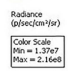

C

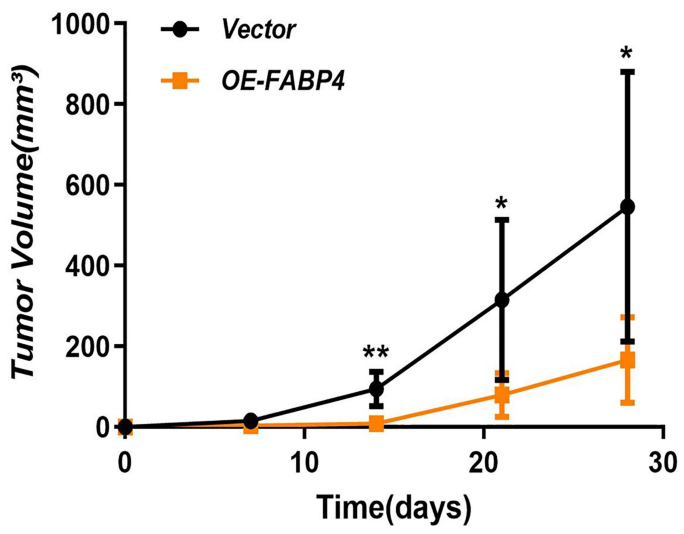

E

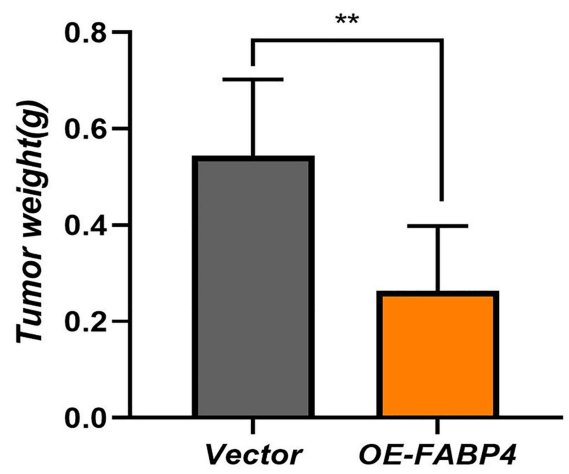

Figure 6 Overexpression of FABP4 suppresses tumor growth in a mouse model. (A) Effects of FABP4 upregulation on tumorigenesis in nude mice evaluated by bioluminescence imaging in vivo. (B) Images were captured immediately after mice were sacrificed, FABP4-overexpressing cell lines were injected into the mice in lower panel image while the mice in the upper panel image were the control group. (C) Tumor volume was measured weekly. (D) Tumors removed from nude mice. (E) Tumor weight. Data are expressed as the mean $\pm \mathrm{SD}$. $* * * \mathrm{P}<0.00 \mathrm{I}, * * \mathrm{P}<0.0 \mathrm{I}, * \mathrm{P}<0.05$. 


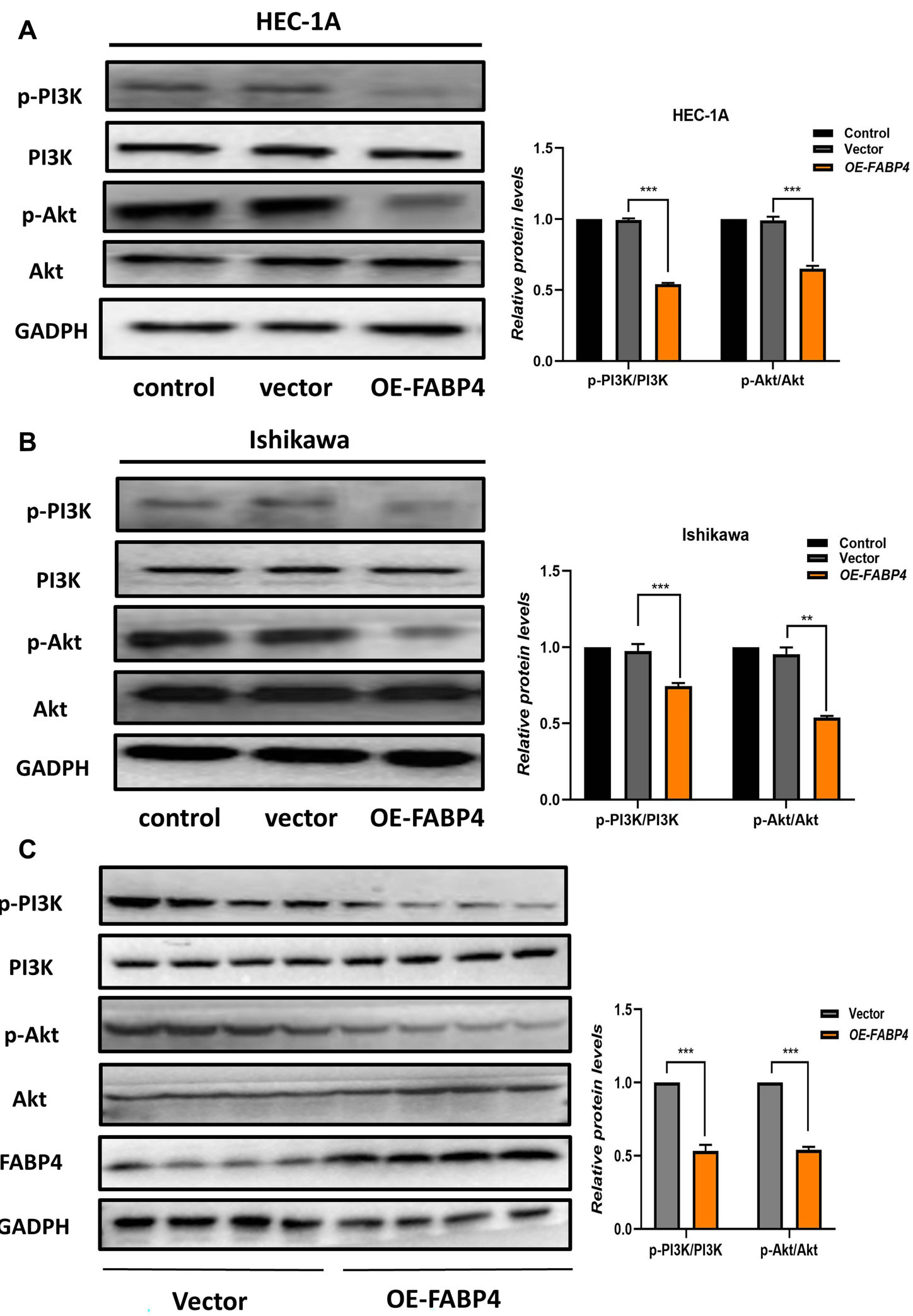

Figure 7 Overexpression of FABP4 suppresses the phosphorylation of PI3K and AKT. (A and B) Protein expression levels of PI3K/Akt pathway in the two cell lines were determined by Western blot analysis. The histogram shows the relative quantification of $\mathrm{p}-\mathrm{PI} \mathrm{KK} / \mathrm{PI} 3 \mathrm{~K}$ and $\mathrm{p}-\mathrm{Akt} / \mathrm{Akt}$. (C) Levels of expression of PI3K/Akt pathway proteins in mice were determined after animals were sacrificed. Data are expressed as the mean $\pm \mathrm{SD}$. $* * * \mathrm{P}<0.00 \mathrm{I}, * * \mathrm{P}<0.0 \mathrm{I}, * \mathrm{P}<0.05$ 
signaling pathway is a classic and extensively studied intracellular signaling pathway in tumorigenesis, which is usually abnormally activated in most cancers. ${ }^{30,31}$ Therefore, certain inhibitors targeting the PI3K/Akt signaling pathway have been regarded as therapeutic potential targets in various cancers. ${ }^{32,33}$ Moreover, recently, Deng et al reported that FABP4 silencing inhibited endoplasmic reticulum (ER) stress-induced cell apoptosis by activating the PI3K/Akt signaling pathway. ${ }^{34}$ Another study by Uehara et al found that exogenous FABP4 promoted human prostate cancer cell progression by activating the PI3K/Akt signaling pathway, independently of binding to fatty acids. ${ }^{35}$ These studies suggested that FABP4 primarily functions upstream of the PI3K/Akt signaling pathway. This suggestion was also confirmed by our experimental results (Figure 6), indicating that overexpression of FABP4 regulated the PI3K/Akt pathway by inhibiting its phosphorylation.

However, interestingly, a recent review proposed another possible explanation for the discrepancies in the function of FABP4. ${ }^{36}$ The author proposed that adipocyte-derived FABP4 enhances tumor progression as mentioned earlier, ${ }^{24,25}$ but peritumor-derived FABP4 impairs tumor progression. ${ }^{29}$ This partly explains why the increase of FABP4 induced by co-culture with fat accelerates the tumor progression, while overexpressing FABP4 in cells that do not express FABP4 themselves weakens tumor progression. In conclusion, gene regulation is a complicated process and is finely tuned in various cellular processes. However, little is known regarding the interacting protein partners and how lipids regulate the subcellular localization of FABP4. Addressing these problems may contribute to a more complete understanding of the function of FABP4.

\section{Conclusions}

We firstly demonstrate that upregulated the expression of FABP4 in EC tissues led to a better outcome. These findings revealed that $\mathrm{FABP} 4$ may be a potential therapeutic target for EC treatment and offer new insights for further investigations of EC pathogenesis.

\section{Abbreviations}

$\mathrm{EC}$, endometrial cancer; FABP4, fatty acid-binding protein 4; TCGA, The Cancer Genome Atlas; PI3K, phosphatidylinositol 3-kinase; Akt, protein kinase B; DMEM, Dulbecco's modified Eagle's medium; FBS, fetal bovine serum; qRT-PCR, quantitative real-time polymerase chain reaction; IHC, immunohistochemistry; HRP, horse radish peroxidase; PMSF, phenylmethylsulfonyl fluoride; SDSPAGE, sodium dodecyl sulfate-polyacrylamide gel electrophoresis; PVDF, polyvinylidene fluoride; CCK-8, cell counting kit-8; FIGO, International Federation of Gynecology and Obstetrics; OS, overall survival; RFS, relapse-free survival.

\section{Data Sharing Statement}

The data that support the findings of this study are available on request from the corresponding author (Dr Ren).

\section{Acknowledgments}

The authors sincerely appreciate the assistance of the Department of Hepatobiliary and Pancreatic Surgery, the First Affiliated Hospital of Zhengzhou University.

\section{Disclosure}

The authors have declared that no competing interest exists.

\section{References}

1. Morice P, Leary A, Creutzberg C, Abu-Rustum N, Darai E. Endometrial cancer. Lancet. 2016;387(10023):1094-1108. doi:10.1016/S0140-6736(15)00130-0

2. Bray F, Ferlay J, Soerjomataram I, Siegel R, Torre L, Jemal A. Global cancer statistics 2018: GLOBOCAN estimates of incidence and mortality worldwide for 36 cancers in 185 countries. CA Cancer J Clin. 2018;68(6):394-424. doi:10.3322/caac.21492

3. Zhang S, Gong TT, Liu FH, Jiang YT, Wu QJ. Global, regional, and national burden of endometrial cancer, 1990-2017: results from the Global Burden of Disease Study, 2017. Front Oncol. 2019;9:1440. doi:10.3389/fonc.2019.01440

4. Urick ME, Bell DW. Clinical actionability of molecular targets in endometrial cancer. Nat Rev Cancer. 2019;19(9):510-521. doi:10.1038/s41568-019-0177-x

5. Arend R, Jones B, Martinez A, Goodfellow PJ. Endometrial cancer: molecular markers and management of advanced stage disease. Gynecol Oncol. 2018;150(3):569-580. doi:10.1016/j. ygyno.2018.05.015

6. Brooks R, Fleming G, Lastra R, et al. Current recommendations and recent progress in endometrial cancer. CA Cancer J Clin. 2019;69 (4):258-279. doi:10.3322/caac. 21561

7. Furuhashi M, Hotamisligil G. Fatty acid-binding proteins: role in metabolic diseases and potential as drug targets. Nat Rev Drug Discov. 2008;7(6):489-503. doi:10.1038/nrd2589

8. Masato F, Shutaro I, Hideki O, Tetsuji M. Lipid chaperones and metabolic inflammation. Int J Inflam. 2011;2011:642612.

9. Hotamisligil G, Bernlohr D. Metabolic functions of FABPs mechanisms and therapeutic implications. Nat Rev Endocrinol. 2015;11 (10):592-605. doi:10.1038/nrendo.2015.122

10. Gharpure K, Pradeep S, Sans M, et al. FABP4 as a key determinant of metastatic potential of ovarian cancer. Nat Commun. 2018;9(1):2923. doi:10.1038/s41467-018-04987-y 
11. Zeng J, Sauter ER, Li B. FABP4: a new player in obesity-associated breast cancer - sciencedirect. Trends Mol Med. 2020;26(4):437-440. doi:10.1016/j.molmed.2020.03.004

12. Jin J, Zhang Z, Zhang S, et al. Fatty acid binding protein 4 promotes epithelial-mesenchymal transition in cervical squamous cell carcinoma through AKT/GSK3 $\beta /$ Snail signaling pathway. Mol Cell Endocrinol. 2018;461:155-164. doi:10.1016/j.mce.2017.09.005

13. Furuhashi M, Saitoh S, Shimamoto K, Miura T. Fatty acid-binding protein 4 (FABP4): pathophysiological insights and potent clinical biomarker of metabolic and cardiovascular diseases. Clin Med Insights Cardiol. 2015;2014(Suppl 3):23-33.

14. Soumaoro L, Uetake H, Higuchi T, Takagi Y, Enomoto M, Sugihara K. Cyclooxygenase-2 expression: a significant prognostic indicator for patients with colorectal cancer. Clin Cancer Res. 2004;10(24):8465-8471. doi:10.1158/1078-0432.CCR-04-0653

15. Livak KJ, Schmittgen $\uparrow$ TD. Analysis of relative gene expression data using real-time quantitative PCR and the 2- $\Delta \mathrm{CT}$ method. Methods. 2001;25(4):402-408. doi:10.1006/meth.2001.1262

16. Berger A, Dao F, Levine D. Angiogenesis in endometrial carcinoma: therapies and biomarkers, current options, and future perspectives. Gynecol Oncol. 2021;160(3):844-850. doi:10.1016/j. ygyno.2020.12.016

17. Onstad MA, Schmandt RE, Lu KH. Addressing the role of obesity in endometrial cancer risk, prevention, and treatment. J Clin Oncol. 2016;34(35):4225. doi:10.1200/JCO.2016.69.4638

18. Rai R, Gong Essel K, Mangiaracina Benbrook D, Garland J, Daniel Zhao Y, Chandra V. Preclinical efficacy and involvement of AKT, mTOR, and ERK kinases in the mechanism of sulforaphane against endometrial cancer. Cancers. 2020;12(5):1273. doi:10.3390/ cancers 12051273

19. Waks A, Winer E. Breast cancer treatment: a review. JAMA. 2019;321(3):288-300. doi:10.1001/jama.2018.19323

20. Pondé N, Brando M, El-Hachem G, Werbrouck E, Piccart M. Treatment of advanced HER2-positive breast cancer: 2018 and beyond. Cancer Treat Rev. 2018;67:10-20. doi:10.1016/j. ctrv.2018.04.016

21. Pennell NA, Arcila ME, Gandara DR, West H. Biomarker testing for patients with advanced non-small cell lung cancer: real-world issues and tough choices. Am Soc Clin Oncol Educ Book. 2019;39 (39):531-542. doi:10.1200/EDBK 237863

22. Stadtmauer E, Mangan P. CAR T-cell therapy: on the verge of breakthrough in many hematologic malignancies. $J$ Adv Pract Oncol. 2017;8(3):228-231. doi:10.6004/jadpro.2017.8.3.3

23. Ockner RK, Manning JA, Poppenhausen RB, Ho WKL. A binding protein for fatty acids in cytosol of intestinal mucosa, liver, myocardium, and other tissues. Science. 1972;177(4043):56-58. doi: $10.1126 /$ science. 177.4043 .56
24. Nieman KM, Kenny HA, Penicka CV, et al. Adipocytes promote ovarian cancer metastasis and provide energy for rapid tumor growth. Nat Med. 2011;17(11):1498. doi:10.1038/nm.2492

25. Nie J, Zhang J, Wang L, et al. Adipocytes promote cholangiocarcinoma metastasis through fatty acid binding protein 4. J Exp Clin Cancer Res. 2017;36(1):183. doi:10.1186/s13046-017-0641-y

26. Santis MLD, Hammamieh R, Das R, Jett M. Adipocyte-fatty acid binding protein induces apoptosis in DU145 prostate cancer cells. J Exp Ther Oncol. 2004;4(2):91-100.

27. Das R, Hammamieh R, Neill R, Melhem M, Jett M. Expression pattern of fatty acid-binding proteins in human normal and cancer prostate cells and tissues. Clin Cancer Res. 2001;7(6):1706-1715.

28. Tölle A, Suhail S, Jung M, Jung K, Stephan C. Fatty acid binding proteins (FABPs) in prostate, bladder and kidney cancer cell lines and the use of IL-FABP as survival predictor in patients with renal cell carcinoma. BMC Cancer. 2011;11(1):302. doi:10.1186/1471-240711-302

29. Zhong C, Zhang X, Ma N, et al. FABP4 suppresses proliferation and invasion of hepatocellular carcinoma cells and predicts a poor prognosis for hepatocellular carcinoma. Cancer Med. 2018;7 (6):2629-2640. doi:10.1002/cam4.1511

30. LoRusso P. Inhibition of the PI3K/AKT/mTOR pathway in solid tumors. J Clin Oncol. 2016;34(31):3803-3815. doi:10.1200/ JCO.2014.59.0018

31. Vara J, Casado E, Castro JD, Cejas P, Belda-Iniesta C, GonzálezBarón M. PI3K/Akt signalling pathway and cancer. Cancer Treat Rev. 2004;30(2):193-204. doi:10.1016/j.ctrv.2003.07.007

32. Wang LL, Zhang L, Cui XF. Downregulation of long noncoding RNA LINC01419 inhibits cell migration, invasion, and tumor growth and promotes autophagy via inactivation of the PI3K/Akt1/mTOR pathway in gastric cancer. Ther Adv Med Oncol. 2019;11:1-16. doi:10.1177/1758835919874651

33. Pei L, Kong Y, Shao C, Yue X, Wang Z, Zhang Z. Heme oxygenase-1 induction mediates chemoresistance of breast cancer cells to pharmorubicin by promoting autophagy via PI3K/Akt pathway. J Cell Mol Med. 2018;22(11):5311-5321. doi:10.1111/jcmm.13800

34. Deng T, Wang Y, Wang C, Yan H. FABP4 silencing ameliorates hypoxia reoxygenation injury through the attenuation of endoplasmic reticulum stress-mediated apoptosis by activating PI3K/Akt pathway. Life Sci. 2019;224:149-156. doi:10.1016/j.1fs.2019.03.046

35. Uehara H, Takahashi T, Oha M, Ogawa H, Izumi K. Exogenous fatty acid binding protein 4 promotes human prostate cancer cell progression. Int $J$ Cancer. 2015;135(11):2558-2568. doi:10.1002/ ijc. 28903

36. McKillop I, Girardi C, Thompson K. Role of fatty acid binding proteins (FABPs) in cancer development and progression. Cell Signal. 2019;62:109336. doi:10.1016/j.cellsig.2019.06.001
OncoTargets and Therapy

\section{Publish your work in this journal}

OncoTargets and Therapy is an international, peer-reviewed, open access journal focusing on the pathological basis of all cancers, potential targets for therapy and treatment protocols employed to improve the management of cancer patients. The journal also focuses on the impact of management programs and new therapeutic agents and protocols on patient perspectives such as quality of life, adherence and satisfaction. The manuscript management system is completely online and includes a very quick and fair peer-review system, which is all easy to use. Visit http://www.dovepress.com/ testimonials.php to read real quotes from published authors. 